\section{Migräne führt nicht zu kognitiven Störungen}

\author{
Bei Patienten mit schwerer Migräne mit Aura und häufigen Attacken kommt \\ es in der Kernspintomografie nicht selten zu Marklagerveränderungen in T2- \\ betonten Schichten und zu klinisch stummen Insulten. Diese könnten theore- \\ tisch zu einer vaskulären Enzephalopathie und einer vaskulären Demenz \\ prädisponieren. Eine Arbeitsgruppe aus Boston ging dieser Frage nach.
}

$\mathrm{D}$ ie Basis dieser Studie bildete die Women's Health Study. Diese große Studie rekrutierte zwischen 1992 und 1995 insgesamt 39.876 Frauen, die im Gesundheitsbereich arbeiteten und 45 Jahre oder älter waren. Die Studie selbst war als eine Therapiestudie zur Wirksamkeit von Acetylsalicylsäure und Vitamin E im Vergleich zu Placebo zur Prävention kardiovaskulärer Ereignisse und maligner Erkrankungen angelegt.

Im Jahr 1998 wurde eine kognitive Substudie gestartet. In diese Studie wurden Frauen aufgenommen, die 65 Jahre oder älter waren. Die insgesamt 6.377
Teilnehmerinnen unterzogen sich einer neuropsychologischen Testbatterie und wurden dann im Abstand von zwei Jahren mindestens zweimal nachuntersucht.

Für diese Analyse standen Daten von 6.349 Frauen zur Verfügung. Davon berichteten 853, unter einer Migräne zu leiden. 195 hatten eine Migräne mit Aura, 248 eine Migräne ohne Aura. 410 Frauen gaben an, in der Vergangenheit eine Migräne gehabt zu haben. Im Vergleich zu Frauen ohne Migräne bestanden keine Differenzen bezüglich kognitiver Funktionen in der Baseline und auch keine Veränderungen über die Zeit hinweg.

\title{
Frühe Menarche erhöht das Risiko eines Ovarialkarzinoms
}

Für die Ätiologie der Ovarialkarzinome sind inzwischen einige Risikofaktoren bekannt. Auch eine Assoziation des Alters bei Menarche mit dem Risiko eines Ovarialkarzinoms wurde jetzt erstmals in einer umfangreichen Metaanalyse bestätigt.

$D^{\prime}$ as Ovarialkarzinom ist der dritthäufigste gynäkologische maligne Tumor; jährlich werden weltweit etwa 225.000 Neuerkrankungen diagnostiziert. Epidemiologische Untersuchungen belegen, dass zunehmendes Lebensalter, eine positive Familienanamnese eines Mamma- oder Ovarialkarzinoms, Kinderlosigkeit, Strahlenexposition und Kontakt zu Asbest Risikofaktoren für diesen Tumor sind. Dagegen reduziert die Einnahme von oralen Kontrazeptiva, stattgehabte Geburten und eine Tubenligation das Risiko. Zwar wird vermutet, dass auch das Alter bei Menarche einen Einfluss auf das spätere Auftreten eines Ovarialkarzinoms haben könnte. Allerdings hatten bisherige Untersuchungen inkonsistente Ergebnisse. Ein weiterer Faktor sind die hormonellen Veränderungen, die in der späten Kindheit und Adoleszenz auftreten und ihrerseits eine Bedeutung in der

Frauen, die unter einer Migräne leiden, unterscheiden sich bezüglich kognitiver Funktionen und Veränderungen kognitiver Funktionen über die Zeit nicht von Frauen, die keine Migräne haben.

P. M. Rist et al. Migraine and cognitive decline among women: prospective cohort study. BMJ 2012;345: e5027. Epub 2012/08/10

Kommentar: Diese Substudie der großen Women's Health Study reproduziert die Ergebnisse anderer großer Register oder weiterer prospektiver Studien, die ebenfalls keinen Zusammenhang zwischen dem Vorliegen einer Migräne und dem Auftreten kognitiver Störungen fanden. Diese Erkenntnisse sind für die klinische Praxis wichtig. Viele Patientinnen sind sehr beunruhigt, wenn sie vom Radiologen mitgeteilt bekommen, dass in ihren Kernspintomografien "weiße Flecken“ zu sehen sind. Dies wird von Allgemeinradiologen häufig als entzündliche Erkrankung des ZNS oder als zerebrale Mikroangiopathie interpretiert.

Prof. Hans-C. Diener

dem Risiko eines Ovarialkarzinoms bestätigte sich auch bei einer Auswertung der meisten Subgruppen. Allerdings betraf dies nur invasive und seröse Borderline-Ovarialkarzinome. Die Autoren folgern aus den Ergebnissen der Metaanalyse, dass das Alter bei der Menarche invers mit dem Risiko eines Ovarialkarzinoms assoziiert ist.

Ätiologie des Ovarialkarzinoms haben. In einer Metaanalyse aller prospektiver Untersuchungen und Fallkontrollstudien, die bis 2012 publiziert wurden, wurde jetzt erstmals die Assoziation zwischen dem Zeitpunkt der Menarche und dem Risiko eines Ovarialkarzinoms untersucht. Insgesamt wurden die Daten von 22 Fall-Kontroll- und 5 Kohortenstudien gepoolt und ausgewertet. Über alle Studien zeigte sich eine inverse statistisch signifikante Assoziation zwischen dem Alter bei Menarche und dem Risiko eines Ovarialkarzinoms (relatives Risiko $=0,85$; 95\%-Konfidenzintervall (KI): 0,75-0,97). Das gepoolte relative Risiko eines Ovarialkarzinoms für eine späte versus frühe Menarche betrug bei den prospektiven Studien 0,89 (95\% KI: 0,76-1,03) und bei den Fall-Kontroll-Studien 0,84 (95\% KI: 0,70-0,99). Die inverse Assoziation zwischen dem Alter bei der Menarche und
Gong TT et al.: Age at menarche and risk of ovarian cancer: A meta-analysis of epidemiological studies. Int J Cancer 2012; doi: 10.1002/ijc.27952. [Epub ahead of print]

Fazit: Die Studie belegt erstmals einen Zusammenhang zwischen dem Beginn der Menarche und dem Risiko eines Ovarialkarzinoms. Die Ergebnisse unterstützen die Hypothese, dass mehr Lebenszeitovulationen mit einem erhöhten Risiko für ein Ovarialkarzinom einhergehen. Ein möglicher Erklärungsansatz hierfür ist das durch die Ovulation verursache Trauma bzw. die mitotische Stimulation auf das ovariale Epithelium. Der heutzutage frühere Beginn der Menarche könnte somit ausgehend von den Folgerungen dieser Studie in den nächsten Jahren zu einer Zunahme des Ovarialkarzinoms führen. Prof. Tino F. Schwarz 